\title{
THE PROBLEM OF CONFUSION IN SURVEYS OF SOURCES
}

\author{
M. RYLE \\ Mullard Radio Astronomy Observatory, Cavendish Laboratory \\ Cambridge, England
}

Dr. Pawsey has asked me to make some general remarks on the problem of confusion when the traces of adjacent radio sources overlap. This is a question that affects all surveys in which the observations are limited by resolving power and not by sensitivity; there is perhaps some misunderstanding about the relative performance of interferometers and pencil-beam systems of similar sizes, and there seems to be an impression that the former are more seriously affected than the latter.

I think this impression may have arisen from the fact that a number of pencil-beam systems have been limited in the detection of weak sources less seriously by confusion than by the use of smaller collecting areas, by the difficulty of distinguishing between sources and irregularities in the general galactic emission, and, in some cases, by the greater influence of man-made interference.

I think it is fairly evident that if we made two systems, one a pencil-beam system, and the other an interferometer using two aerials of equal size to the one of the pencil-beam system, then our records in either case would be made up of contributions from sources in the same area of sky. At first sight, therefore, it is likely that there will be very little difference in the number of sources we can reliably detect. In fact the interferometer would offer us some advantages, since for every beamwidth we can measure a phase in addition to the amplitude; if now we want to test whether a given possible configuration of sources fits the observations we have twice as much information with which to test our model, and the chances of a serious misinterpretation are reduced.

Now how does confusion affect our observations? This depends very much on what we want to do :

1. If we are interested in the very accurate determination of the positions of sources-for example in the search for optical identifications-or if we wish to find the flux density of a source to say 5 per cent for spectral work, we may have to ensure that the wanted source produces a record twenty times the size of any other; this may only allow us to observe one source per 100 beamwidths.

2. If we are making a general survey of sources, we may be happy with flux densities accurate to 25 per cent, and we may be able to work to a level of one source per 10 beamwidths.

3. If we are only concerned with the distribution of sources in space and are not interested in their individual positions, we can use a different approach 
-as shown by Scheuer [1]-and examine the statistical properties of the record deflection. This method allows us to work down to about one source per beamwidth.

How do we know the magnitude of the effects of confusion? In the case of an interferometer, this is fairly easy: the record at any time may be regarded as the vector sum of the trace from the wanted source and that caused by the sum of all the confusing sources. These two vectors are equally likely to occur with any phase angle, and the magnitude of the confusing vector may be derived directly by plotting a histogram (Fig. 1) of the record amplitude read off a long section of the record.

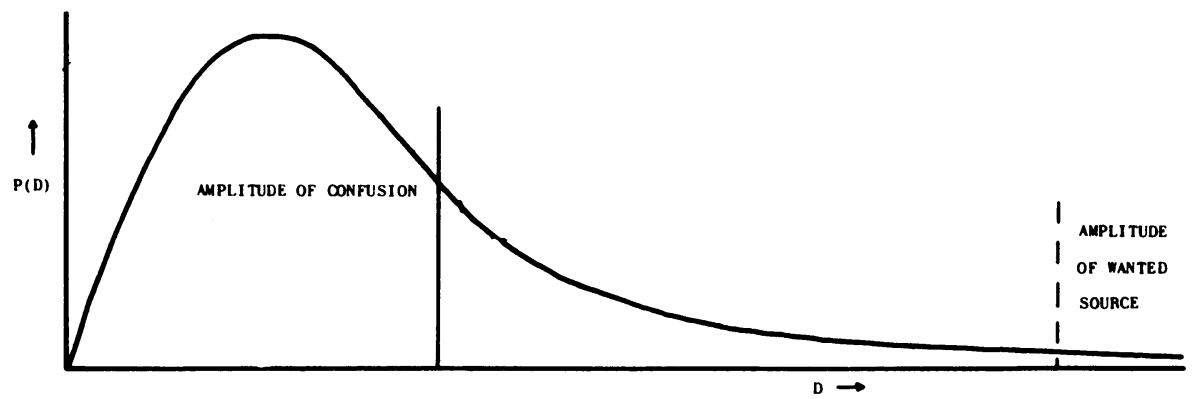

FIG. 1. Histogram of the record amplitude $D$ derived from a sample of the record. The area to the right of the full line gives the probability of the "confusion" exceeding a given amplitude.

The probability of the record's having a given amplitude say 20 per cent of that produced by the wanted source tells us the probability of our having a 20 per cent error in flux or a positional error corresponding to $1 / 5$ radian in the angular periodicity of the interference pattern (Fig. 2).

Similar methods could be applied to observations with pencil-beam systems,

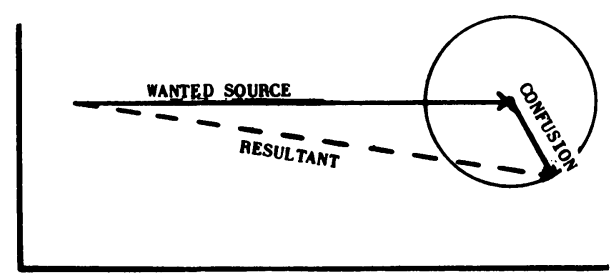

FIG. 2. The observed record will usually be composed of the addition of the wanted signal and a "confusion" vector; by relating the amplitude of the wanted signal to the histogram of Fig. 1, the probability that the error in flux density or position exceeds a given value may be computed. only here there are practical difficulties caused by the uncertainty in the base level owing to the galactic structure; in principle, however, we can estimate with any system the probability that confusing sources introduce errors of a given magnitude.

As I have already mentioned, the study of the "fully confused" record offers important possibilities for investigating the spatial distribution of sources in systems where the sensitivity is adequate.

\section{REFERENCE}

[1] Scheuer, P. A. G. Proc. Camb. Phil. Soc. 53, 764, 1957. 